\title{
A current algebra approach to the equilibrium classical statistical mechanics and its applications*
}

\author{
N.N. Bogolubov (Jr.) 1 , A.K. Prykarpatsky 23 \\ 1 V.A. Steklov Mathematical Institute of RAN, Moscow, Russian Federation \\ 2 AGH University of Science and Technology, 30-059 Krakow, Poland \\ ${ }^{3}$ Ivan Franko State Pedagogical University, Drohobych, Ukraine
}

Received October 11, 2012

\begin{abstract}
The non-relativistic current algebra approach is analyzed subject to its application to studying the distribution functions of many-particle systems at the temperature equilibrium and their stability properties. We show that the classical Bogolubov generating functional method is a very effective tool for constructing the irreducible current algebra representations and the corresponding different generalized measure expansions including collective variables transform. The effective Hamiltonian operator construction and its spectrum peculiarities subject to the stability of equilibrium many-particle systems are discussed.
\end{abstract}

Key words: current algebra, Bogolubov generating functional, collective variables representation, Hamiltonian operator reconstruction

PACS: 73.21.Fg, 73.63. $\mathrm{Hs}$, 78.67.De

\section{Introduction}

It is well known [1-4] that a complete physical theory, both relativistic and non-relativistic, can be described entirely in terms of current algebra operators, such as current densities, rather than in terms of canonical field operators, which is motivated by the fact that the current densities are physically observable quantities contrary to the canonical non-observable field operators. Moreover, the current algebra approach appeared to be also very effective in studying both quantum and classical statistical problems of many-particle systems by means of the Bogolubov generating functional, whose mathematical structure became a fruitful source of many approximation methods in modern statistical physics. Amongst them it is necessary to mention a very powerful collective variables transform suggested firstly by D. Bohm [5] and deeply developed by N. Bogolubov [6], D. Zubarev [7] and I. Yukhnovskii [8]. This transform has been reanalyzed in terms of the current algebra approach for classical many-particle systems in [9-11], where there was constructed a corresponding Bogolubov generating functional of distributions as a mathematical expectation of an infinite hierarchy of the non-interacting many-particle systems embedded into an external oscillatory potential field, with respect to a suitably defined infinite divisible Gauss type measure.

Based on these results and making use of some additional properties of the corresponding functional equations for the Bogolubov generating functional of many-particle distribution functions we have constructed, for the case of classical statistical mechanics, a new operator representation for an effective Hamiltonian operator defined in a suitable Hilbert space, whose ground state energy peculiarities make it possible to conceive the physical nature of the related phase transitions and to describe the behavior of multi-particle distribution functions.

\footnotetext{
* Authors devote this work to their friend and colleague, a smart phase transitions researcher Professor Mykhailo Kozlovskii on the occasion of his 60 -years anniversary.
} 


\section{Non-relativistic quantum and statistical mechanics: the current alge- bra approach}

We assume a non-relativistic spinless many particle system of density $\bar{\rho} \in \mathbb{R}_{+}$to be described by means of the non-relativistic quantum Hamiltonian operator

$$
\mathbf{H}:=\frac{\hbar^{2}}{2 m} \int_{\mathbb{R}^{3}} \mathrm{~d}^{3} x\left\langle\nabla \psi^{+}(x), \nabla \psi(x)\right\rangle+\frac{1}{2} \int_{\mathbb{R}^{3}} \mathrm{~d}^{3} x \int_{\mathbb{R}^{3}} \mathrm{~d}^{3} y W(x, y) \psi^{+}(x) \psi^{+}(y) \psi(y) \psi(x)
$$

acting in a suitable Fock space $\Phi$, here we have denoted by $\langle\cdot, \cdot\rangle$ the standard scalar product in the Euclidean space $\mathbb{R}^{3}, W: \mathbb{R}^{3} \times \mathbb{R}^{3} \rightarrow \mathbb{R}$ is a translation invariant interaction potential, and creation $\psi^{+}(x): \Phi \rightarrow \Phi, x \in \mathbb{R}^{3}$ and annihilation operators $\psi(y): \Phi \rightarrow \Phi, y \in \mathbb{R}^{3}$, satisfy the standard canonical commutation relationships:

$$
\left[\psi(y), \psi^{+}(x)\right]=\delta(x-y), \quad\left[\psi^{+}(x), \psi^{+}(y)\right]=0=[\psi(x), \psi(y)] .
$$

The current algebra representation of the Hamiltonian operator (2.1) is based on the following selfadjoint density operators: particle number density

$$
\rho(x):=\psi^{+}(x) \psi(x)
$$

and current density

$$
J(x):=\frac{1}{2 \mathrm{i}}\left[\psi^{+}(x) \nabla \psi(x)-\nabla \psi^{+}(x) \psi(x)\right]
$$

at any point $x \in \mathbb{R}^{3}$, satisfying the well known classical current Lie algebra commutator relationships:

$$
\left[\rho\left(f_{1}\right), \rho\left(f_{2}\right)\right]=0, \quad[\rho(t), J(g)]=\mathrm{i} \rho(\langle g, \nabla f\rangle), \quad\left[J\left(g_{1}\right), J\left(g_{2}\right)\right]=\mathrm{i} J\left(\left[g_{2}, g_{1}\right]\right),
$$

where we have defined the smeared [3, 12, 13] density operators

$$
\rho(f):=\int_{\mathbb{R}^{3}} \mathrm{~d}^{3} x \rho(x) f(x), \quad J(g):=\int_{\mathbb{R}^{3}} \mathrm{~d}^{3} x\langle g(x), J(x)\rangle
$$

for any Schwartz functions $f \in \mathscr{S}\left(\mathbb{R}^{3} ; \mathbb{R}\right)$ and $g \in \mathscr{S}\left(\mathbb{R}^{3} ; \mathbb{R}^{3}\right)$ and $\left[g_{2}, g_{1}\right]:=\left\langle g_{2}, \nabla\right\rangle g_{1}-\left\langle g_{1}, \nabla\right\rangle g_{2}$ for any $g_{1}, g_{2} \in J\left(\mathbb{R}^{3} ; \mathbb{R}^{3}\right)$.

The following proposition characterizes [1, 3, 13, 14] the current Lie algebra (2.4) from the group representation theory.

The exponential current operators are as follows:

$$
U(f):=\exp [\mathrm{i} \rho(f)], \quad V\left(\varphi_{t}^{g}\right):=\exp [\mathrm{i} t J(g)],
$$

where $\mathrm{d} \varphi_{t}^{g}(x) / \mathrm{d} t:=g \circ \varphi_{t}^{g}(x)$ and $g \circ \varphi_{t}^{g}(x):=g\left[\varphi_{t}^{g}(x)\right]$ for any $t \in \mathbb{R}$ and $x \in \mathbb{R}^{3}$, satisfy the current group relationships

$$
U\left(f_{1}\right) U\left(f_{2}\right)=U\left(f_{1}+f_{2}\right), \quad V(\varphi) U(f)=U(f \circ \varphi) V(\varphi), \quad V\left(\varphi_{1}\right) \circ V\left(\varphi_{2}\right)=V\left(\varphi_{2} \circ \varphi_{1}\right)
$$

for the semi-simple product $G:=\mathscr{S} \rtimes \operatorname{Diff}\left(\mathbb{R}^{3}\right)$ and the abelian Schwartz group $\mathscr{S}\left(\mathbb{R}^{3} ; \mathbb{R}\right)$, where $f_{1}, f_{2}$ and $f \in \mathscr{S}\left(\mathbb{R}^{3} ; \mathbb{R}\right), \varphi_{1}, \varphi_{2}$ and $\varphi \in \operatorname{Diff}\left(\mathbb{R}^{3}\right)$. The latter appeared to be very important in constructing the corresponding group $G=\mathscr{S} \rtimes \operatorname{Diff}\left(\mathbb{R}^{3}\right)$ representations in suitable Hilbert spaces and their physical interpretation as a classical generating Bogolubov functional [6, 11, 13, 14] for the corresponding many-particle distribution functions.

The Hamiltonian operator 2.1 permits the following current algebra representation

$$
\mathbf{H}=\frac{\hbar^{2}}{8 m} \int_{\mathbb{R}^{3}} \mathrm{~d}^{3} x\left\langle K^{+}(x), \rho^{-1}(x) K(x)\right\rangle+\frac{1}{2} \int_{\mathbb{R}^{3}} \mathrm{~d}^{3} x \int_{\mathbb{R}^{3}} \mathrm{~d}^{3} y W(x, y): \rho(x) \rho(y):,
$$


where, by definition,

$$
K(x):=\nabla \rho(x)+2 \mathrm{i} J(x)
$$

for any $x \in \mathbb{R}^{3}$ and the normal ordering [15, 16] acts as

$$
: \rho\left(x_{1}\right) \rho\left(x_{2}\right) \ldots \rho\left(x_{n}\right):=\prod_{j=1}^{n}\left[\rho\left(x_{j}\right)-\sum_{k=1}^{j-1} \delta\left(x_{j}-x_{k}\right)\right]
$$

for arbitrary $x_{j} \in \mathbb{R}^{3}, j=\overline{1, n}, n \in \mathbb{Z}_{+}$.

The current group $G=\mathscr{S} \rtimes \operatorname{Diff}\left(\mathbb{R}^{3}\right)$, as is well known, possesses many different irreducible unitary representations in suitable Hilbert spaces. In particular, in the standard $N$-particle Hilbert space $\mathscr{H}^{(N)}:=$ $L_{2}^{\text {(sym) }}\left(\mathbb{R}^{3 N} ; \mathbb{C}\right)$ for an arbitrary but fixed $N \in \mathbb{Z}_{+}$the particle density operator acts as

$$
\rho(f) \omega=\left[\sum_{j=1}^{N} f\left(x_{j}\right)\right] \omega
$$

and the current density operator acts as

$$
J(g) \omega=\frac{1}{2 \mathrm{i}} \sum_{j=1}^{N}\left[\left\langle g\left(x_{j}\right), \nabla_{j}\right\rangle+\left\langle\nabla_{j}, g\left(x_{j}\right)\right\rangle\right] \omega
$$

for any $f \in \mathscr{S}\left(\mathbb{R}^{3} ; \mathbb{R}\right), g \in \mathscr{S}\left(\mathbb{R}^{3} ; \mathbb{R}^{3}\right)$ and arbitrary vector $\omega \in \hat{H}^{(N)}$.

In the general case, the current group (2.7) possesses many different irreducible unitary representations in suitable Hilbert spaces $\mathscr{H}$, which can be written down as

$$
\mathscr{H}=\int_{\mathscr{S}^{\prime}}^{\oplus} \mathrm{d} \mu(F) \mathscr{H}_{F},
$$

where $\mu: 2^{\mathscr{S}^{\prime}} \rightarrow \mathbb{R}_{+}$is some cylindrical measure on the generalized space $\mathscr{S}^{\prime}:=\mathscr{S}^{\prime}\left(\mathbb{R}^{3} ; \mathbb{R}\right), \mathcal{H}_{F}$ are marked by elements $F \in \mathscr{S}^{\prime}\left(\mathbb{R}^{3} ; \mathbb{R}\right)$ complex linear spaces, which for many physical applications [3, 4, 13] are one-dimensional. In the case $\operatorname{dim} \mathscr{H}_{F}=1$, one obtains from $(2.13)$ that $\mathscr{H} \simeq L_{2}^{(\mu)}\left(\mathscr{S}^{\prime} ; \mathbb{C}\right)$. Now, if an element $\omega(F) \in \mathscr{H}$ is taken arbitrarily, from (2.7) one easily follows that

$$
\begin{aligned}
& U(f) \omega(F)=\exp [\mathrm{i}(F, f)] \omega(F), \\
& V(\varphi) \omega(F)=\chi_{\varphi}(F) \omega\left(\varphi^{*} F\right)\left[\frac{\mathrm{d} \mu\left(\varphi^{*} F\right)}{\mathrm{d} \mu(F)}\right]^{1 / 2},
\end{aligned}
$$

where, by definition, $\left(\varphi^{*} F, f\right):=(F, f \circ \varphi), \mathrm{d} \mu\left(\varphi^{*} F\right) / \mathrm{d} \mu(F)$ is the standard Radon-Nykodym derivative of the measure $\mu\left(\varphi^{*} F\right)$ with respect to the measure $\mu\left(\varphi^{*} F\right)$ and $\chi_{\varphi}(F)$ is a complex-valued factor of a unit norm, referred to as the co-cycle, satisfying the relationship

$$
\chi_{\varphi_{2}}(F) \chi_{\varphi_{1}}\left(\varphi_{2}^{*} F\right)=\chi_{\varphi_{1} \circ \varphi_{2}}(F)
$$

for any $\varphi_{1}, \varphi_{2} \in \operatorname{Diff}\left(\mathbb{R}^{3}\right)$ and arbitrary point $F \in \mathscr{S}^{\prime}\left(\mathbb{R}^{3} ; \mathbb{R}\right)$.

Now, based on the expression (2.14), one can define the following functional

$$
\mathscr{L}(f):=(\Omega, \exp [\mathrm{i} \rho(f)] \Omega)
$$

for the physical ground state vector $\Omega \in \mathscr{H}$ of the suitably renormalized [1, 11, 13, 14] Hamiltonian operator (2.8):

$$
\Omega:=\arg \inf _{\omega \in \mathcal{H},\|\omega\|=1} \frac{(\omega, \mathbf{H} \omega)}{(\omega, \mathbf{N} \omega)} .
$$

This, in particular, means that the Hilbert space representation of the current group $G=\mathscr{S} \rtimes \operatorname{Diff}\left(\mathbb{R}^{3}\right)$ is chosen in such a way that the corresponding Hamiltonian operator $(2.8)$ is bounded from below, thereby 
realizing a respectively stable physical many-particle system. Taking this into account one can put, without loss of generality, that the Hamiltonian operator (2.8) and its ground state vector (2.17) satisfy the equivalent conditions

$$
\mathbf{H} \Omega=0, \quad(\Omega, \rho(x) \Omega)=\bar{\rho}>0 .
$$

Now, we can interpret the functional (2.16) as a generating functional of the current group $G=\mathscr{S} \rtimes$ $\operatorname{Diff}\left(\mathbb{R}^{3}\right)$ irreducible representations $[9,13,14]$ in the physically proper Hilbert space $\mathscr{H}$. This is based on the following theorem [1] owing to H. Araki.

Theorem 2.1. A functional $\mathscr{L}: G \rightarrow \mathbb{C}$ generates a unitary representation of the group $G$ if and only if there exists a unitary continuous representation $\pi: G \rightarrow$ Aut $\mathscr{H}$ with a cyclic vector $\Omega \in \mathscr{H}$ satisfying the condition

$$
\mathscr{L}(a)=(\Omega, \pi(a) \Omega), \quad \mathscr{H}=\operatorname{span}\{a \Omega \in \mathscr{H}: a \in G\}
$$

for any $a \in G$.

Having applied theorem 2.1 to the functional (2.16), we derive that by means of constructing suitable generating functionals subject to the given Hamiltonian operator (2.8) one can find the corresponding operator representations of the current group $G=\mathscr{S} \rtimes \operatorname{Diff}\left(\mathbb{R}^{3}\right)$ and vice versa.

\section{The current algebra representations and the Hamiltonian operator reconstruction}

Based on the relationships (2.14) and the generating functional expression (2.16), one can easily calculate that

$$
\mathscr{L}(f)=\int_{\mathscr{S}^{\prime}} \exp \{\mathrm{i}(F, f)\} \mathrm{d} \mu(F)
$$

for some suitably determined quasi-invariant measure $\mu: 2^{\mathscr{S}^{\prime}} \rightarrow \mathbb{R}_{+}$, that is an ergodic measure with respect to the diffeomorphism group $\operatorname{Diff}\left(\mathbb{R}^{3}\right)$ : for any $\operatorname{Diff}\left(\mathbb{R}^{3}\right)$-invariant set $Q \subset \mathscr{S}^{\prime}\left(\mathbb{R}^{3} ; \mathbb{R}\right)$ either $\mu(Q)=0$ or $\mu\left(\mathscr{S}^{\prime} \backslash Q\right)=0$. As a result of 3.1 one finds, as an example, that the standard quantum mechanical $N$ particle representation of the current group $G=\mathscr{S} \rtimes \operatorname{Diff}\left(\mathbb{R}^{3}\right)$ is described [1, 9, 13] by the generalized singular measure

$$
\mathrm{d} \mu(F)=\Omega^{*} \Omega \prod_{j=1}^{N} \mathrm{~d}^{3} x_{j} \delta\left(F-\sum_{k=1}^{N} \delta\left(x-x_{k}\right)\right),
$$

whose support supp $\mu=\left\{F \in \mathscr{S}^{\prime}\left(\mathbb{R}^{3} ; \mathbb{R}\right): F=\sum_{k=1}^{N} \delta\left(x-x_{k}\right)\right\}$.

Consider now the generating functional $(2.16)$ and observe that the following quantities

$$
\begin{aligned}
F_{n}\left(x_{1}, x_{2}, \ldots, x_{n}\right) & :=\left(\Omega,: \rho\left(x_{1}\right) \rho\left(x_{2}\right) \ldots \rho\left(x_{n}\right): \Omega\right) \\
& =: \frac{1}{\mathrm{i}} \frac{\delta}{\delta f\left(x_{1}\right)} \frac{1}{\mathrm{i}} \frac{\delta}{\delta f\left(x_{2}\right)} \ldots \frac{1}{\mathrm{i}} \frac{\delta}{\delta f\left(x_{n}\right)}:\left.\mathscr{L}(f)\right|_{f=0}
\end{aligned}
$$

for arbitrary $n \in \mathbb{Z}_{+}$represent the $n$-particle distribution functions of the quantum mechanical manyparticle system with the Hamiltonian (2.8), or equivalently, the functional (2.16) is respectively, the Bogolubov generating functional of many-particle distribution functions. Since the essence of the Bogolubov generating functional is held in the correspondingly derived [6] functional equation, we proceed now to determine its exact analytical form taking into account the structure of the related current group $G=\mathscr{S} \rtimes \operatorname{Diff}\left(\mathbb{R}^{3}\right)$ representation in a suitable Hilbert space $\mathscr{H}$.

Following the standard operator construction, suggested in [13, 14], one can define a selfadjoint operator $A(x ; \rho): \mathscr{H} \rightarrow \mathscr{H}, x \in \mathbb{R}^{3}$, by means of the relationships

$$
K(g) \Omega=A(g ; \rho) \Omega,
$$


satisfied for any $g \in \mathscr{S}\left(\mathbb{R}^{3} ; \mathbb{R}^{3}\right)$, where we put, by definition,

$$
K(g):=\int_{\mathbb{R}^{3}} \mathrm{~d}^{3} x\langle g(x), K(x)\rangle, \quad A(g ; \rho):=\int_{\mathbb{R}^{3}} \mathrm{~d}^{3} x\langle g(x), A(x ; \rho)\rangle .
$$

To proceed further we need an important proposition concerning the matrix elements of the operators $J(g)$ and $\mathbf{H}: \not{H} \rightarrow \mathscr{H}$ for any $g \in \mathscr{S}\left(\mathbb{R}^{3} ; \mathbb{R}^{3}\right)$.

Proposition 3.1. Let $\left\{\tilde{f}=\exp [\mathrm{i} \rho(f)] \Omega \in \mathscr{H}: f \in \mathscr{S}\left(\mathbb{R}^{3} ; \mathbb{R}\right)\right\}$ be the set of vectors dense in the Hilbert space $\not{H}$ owing to the Araki's theorem 2.1 Then, the following scalar product expressions

$$
\begin{aligned}
\left(\tilde{f}_{1}, J(g) \tilde{f}_{2}\right) & =\left(\tilde{f}_{1}, \rho\left(\left\langle g, \nabla\left(\tilde{f}_{1}+\tilde{f}_{2}\right)\right\rangle\right) \tilde{f}_{2}\right), \\
\left(\tilde{f}_{1}, \mathbf{H} \tilde{f}_{2}\right) & =\frac{\hbar^{2}}{8 m}\left(\tilde{f}_{1}, \rho\left(\left\langle\nabla \tilde{f}_{1}, \nabla \tilde{f}_{2}\right\rangle\right) \tilde{f}_{2}\right)
\end{aligned}
$$

hold for all $f_{1}, f_{2} \in \mathscr{S}\left(\mathbb{R}^{3} ; \mathbb{R}\right)$.

Based now on simple enough but slightly cumbersome calculations, one can derive the following renormalized Hamiltonian operator expression:

$$
\tilde{\mathbf{H}}=\frac{\hbar^{2}}{8 m} \int_{\mathbb{R}^{3}} \mathrm{~d}^{3} x\left\langle\tilde{K}^{+}(x ; \rho), \rho^{-1}(x) \tilde{K}(x ; \rho)\right\rangle,
$$

where, by definition, the operator

$$
\tilde{K}(x ; \rho):=K(x)-A(x ; \rho)
$$

satisfies the condition

$$
\tilde{K}(x ; \rho) \Omega=0
$$

for all $x \in \mathbb{R}^{3}$. Now, making use of (3.6), we can rewrite the defining condition (3.9) in the following functional equation form:

$$
\left[\nabla_{x}-\nabla f(x)\right] \frac{1}{\mathrm{i}} \frac{\delta \mathscr{L}(f)}{\delta f(x)}=A(x ; \delta) \mathscr{L}(f),
$$

where we put for any $x \in \mathbb{R}^{3}$

$$
A(x ; \delta):=\left.A(x ; \rho)\right|_{\rho=\frac{1}{\mathrm{i}} \frac{\delta}{\delta f}} .
$$

Similarly, one can calculate the matrix element values for the renormalized Hamiltonian operator (3.7) subject to the irreducible cyclic representation of the current group $G=\mathscr{S} \rtimes \operatorname{Diff}\left(\mathbb{R}^{3}\right)$ :

$$
\left(\tilde{f}_{1}, \tilde{\mathbf{H}} \tilde{f}_{2}\right)=\frac{\hbar^{2}}{8 m}\left(\tilde{f}_{1}, \rho\left(\left\langle\nabla \tilde{f}_{1}, \nabla \tilde{f}_{2}\right\rangle\right) \tilde{f}_{2}\right)=\left(\tilde{f}_{1}, \mathbf{H} \tilde{f}_{2}\right)
$$

for all $f_{1}, f_{2} \in \mathscr{S}\left(\mathbb{R}^{3} ; \mathbb{R}\right)$, meaning that two current algebra operator representations (2.8) and (3.7) of the initial Hamiltonian operator (2.1), defined in the canonical Fock space, are physically completely equivalent.

\section{The generating Bogolubov functional equation for the temperature equilibrium states}

Assume that a classical many-particle system is at a bounded inverse temperature $\beta \in \mathbb{R}_{+}$and its Gibbs statistical operator

$$
\mathscr{P}:=\frac{\exp (-\beta \mathbf{H})}{\operatorname{tr} \exp (-\beta \mathbf{H})},
$$

where "tr" means the standard trace-operation well determined on the ideal of nuclear operators in a Hilbert space $\mathscr{H}$, in which the corresponding irreducible unitary representation of the current group 
$G=\mathscr{S} \rtimes \operatorname{Diff}\left(\mathbb{R}^{3}\right)$ is realized. To determine it analytically, we define the Bogolubov generating functional of many-particle distribution functions as

$$
\mathscr{L}(f):=\operatorname{tr}\{\mathscr{P} \exp [\mathrm{i} \rho(f)]\}
$$

for any $f \in \mathscr{S}\left(\mathbb{R}^{3} ; \mathbb{R}\right)$. having imposed on the functional 4.2 the Araki's conditions of theorem 2.1 we can easily derive that there exists $[1,3,4,11,14]$ an effective normalized cyclic vector $\Omega_{\beta} \in \mathscr{H}_{\beta}$, naturally corresponding to the effective Hamilton operator

$$
\tilde{\mathbf{H}}_{\beta}:=\frac{\hbar^{2}}{8 m} \int_{\mathbb{R}^{3}} \mathrm{~d}^{3} x\left\langle\tilde{K}_{\beta}^{+}(x ; \rho), \rho^{-1}(x) \tilde{K}_{\beta}(x ; \rho)\right\rangle,
$$

such that

$$
\mathscr{L}(f)=\left(\Omega_{\beta}, \exp [\mathrm{i} \rho(f)] \Omega_{\beta}\right)
$$

where we have put, by definition,

$$
\tilde{K}_{\beta}(x ; \rho):=K(x)-A_{\beta}(x ; \rho), \quad K(x) \Omega_{\beta}:=A_{\beta}(x ; \rho) \Omega_{\beta}
$$

for any $x \in \mathbb{R}^{3}$. As a result of the definition [4.2) and relationships [4.5), one easily finds [9], as the Planck constant $\hbar \rightarrow 0$, that

$$
\mathscr{L}(f)=\frac{\exp [-\beta W(\delta)] \mathscr{L}_{0}(f)}{\left.\exp [-\beta W(\delta)] \mathscr{L}_{0}(f)\right|_{f=0}},
$$

where $\mathscr{L}_{0}(f), f \in \mathscr{S}\left(\mathbb{R}^{3} ; \mathbb{R}\right)$, is the generating functional for the noninteracting equilibrium many-particle system and, by definition, we put

$$
W(\delta):=\left.W(\rho)\right|_{\rho=\frac{1}{\mathrm{i}} \frac{\delta}{\delta f}} .
$$

Similarly to the above reasonings one also finds that the generating functional [4.6) satisfies [6, 9] the Bogolubov type functional equation

$$
\left[\nabla_{x}-\nabla f(x)\right] \frac{1}{\mathrm{i}} \frac{\delta \mathscr{L}(f)}{\delta f(x)}=A_{\beta}(x ; \delta) \mathscr{L}(f),
$$

where the corresponding operator $A_{\beta}(x ; \rho): \mathscr{H}_{\beta} \rightarrow \mathscr{H}_{\beta}$ for any $x \in \mathbb{R}^{3}$ linearly depends on the binary interparticle interaction potential $W: \mathbb{R}^{3} \times \mathbb{R}^{3} \rightarrow \mathbb{R}$.

Hence, one easily infers that the generating functional $\mathscr{L}_{0}(f), f \in \mathscr{S}\left(\mathbb{R}^{3} ; \mathbb{R}\right)$ for the unitary representation of the current group $G=\mathscr{S} \rtimes \operatorname{Diff}\left(\mathbb{R}^{3}\right)$ satisfies the reduced functional equation

$$
\left[\nabla_{x}-\nabla f(x)\right] \frac{1}{\mathrm{i}} \frac{\delta \mathscr{L}_{0}(f)}{\delta f(x)}=0
$$

whose general non-normalized solution equals the integral

$$
\mathscr{L}_{0}(f)=\int_{\mathbb{R}} \mathrm{d} \mu_{\beta}(z) \exp \left(z \int_{\mathbb{R}^{3}} \mathrm{~d}^{3} x\{\exp [\mathrm{i} f(x)]-1\}\right)
$$

with respect to some Radon measure $\mu_{\beta}: 2^{\mathbb{R}} \rightarrow \mathbb{R}$ on the real axis $\mathbb{R}$. Thus, submitting (4.10) into (4.6), we obtain from (4.8) that for any $x \in \mathbb{R}$

$$
A_{\beta}(x ; \rho)=-\beta \int_{\mathbb{R}^{3}} \mathrm{~d}^{3} y \nabla_{x} W(x, y): \rho(x) \rho(y):
$$

and, respectively,

$$
\left[\nabla_{x}-\nabla f(x)\right] \frac{1}{\mathrm{i}} \frac{\delta \mathscr{L}(f)}{\delta f(x)}=-\beta \int_{\mathbb{R}^{3}} \mathrm{~d}^{3} y \nabla_{x} W(x, y): \frac{1}{\mathrm{i}} \frac{\delta}{\delta f(x)} \frac{1}{\mathrm{i}} \frac{\delta}{\delta f(y)}: \mathscr{L}(f) .
$$


The functional equation (4.12), being well known long ago owing to the classical results of Bogolubov [6], makes it possible, using the current algebra approach, to interpret it as an equation for the generating functional of irreducible current group $G=\mathscr{S} \rtimes \operatorname{Diff}\left(\mathbb{R}^{3}\right)$ representations in a suitable Hilbert space $\mathscr{H}_{\beta}$ with a cyclic vector $\Omega_{\beta} \in \mathscr{H}_{\beta}$ being the ground state vector for a respectively renormalized "effective" positive definite Hamiltonian operator (4.3) and satisfying the conditions (4.5). This gives rise to the following canonical current algebra representation of the Hamiltonian operator (4.3):

$$
\begin{aligned}
\tilde{\mathbf{H}}_{\beta}= & \frac{\hbar^{2}}{8 m} \int_{\mathbb{R}^{3}} \mathrm{~d}^{3} x\left\langle K^{+}(x), \rho^{-1}(x) K(x)\right\rangle \\
& +\sum_{n-2 \in \mathbb{Z}_{+}} \frac{1}{n !} \int_{\mathbb{R}^{3}} \mathrm{~d}^{3} x_{1} \int_{\mathbb{R}^{3}} \mathrm{~d}^{3} x_{2} \ldots \int_{\mathbb{R}^{3}} \mathrm{~d}^{3} x \tilde{W}_{\beta}^{(n)}\left(x_{1}, x_{2}, \ldots, x_{n}\right): \rho\left(x_{1}\right) \rho\left(x_{2}\right) \ldots \rho\left(x_{n}\right):,
\end{aligned}
$$

where the effective inter-particle potentials $\tilde{W}_{\beta}^{(n)}: \underset{j=1}{\times} \mathbb{R}_{j}^{3} \rightarrow \mathbb{R}, n-2 \in \mathbb{Z}_{+}$, non-locally depend on the initial interparticle potential $W: \mathbb{R}^{3} \times \mathbb{R}^{3} \rightarrow \mathbb{R}$ and on the inverse temperature parameter $\beta \in \mathbb{R} \div$.

The Hamiltonian operator 4.13 can be respectively transformed to the canonical form

$$
\begin{aligned}
\tilde{\mathbf{H}}_{\beta}= & \frac{\hbar^{2}}{2 m} \int_{\mathbb{R}^{3}} \mathrm{~d}^{3} x\left\langle\nabla \psi^{+}(x), \nabla \psi(x)\right\rangle \\
& +\sum_{n-2 \in \mathbb{Z}_{+}} \frac{1}{n !} \int_{\mathbb{R}^{3}} \mathrm{~d}^{3} x_{1} \int_{\mathbb{R}^{3}} \mathrm{~d}^{3} x_{2} \ldots \int_{\mathbb{R}^{3}} \mathrm{~d}^{3} x \tilde{W}_{\beta}^{(n)}\left(x_{1}, x_{2}, \ldots, x_{n}\right) \\
& \times: \psi^{+}\left(x_{1}\right) \psi\left(x_{1}\right) \psi^{+}\left(x_{2}\right) \psi\left(x_{2}\right) \ldots \psi^{+}\left(x_{n}\right) \psi\left(x_{n}\right):
\end{aligned}
$$

acting in the standard Fock space $\Phi$. The latter can be used for determining the related canonical $N$ particle representation of the Hamiltonian (4.14) by means of the following defining relationship:

$$
\begin{aligned}
& \tilde{\mathbf{H}}_{\beta}\left(\sum_{n \in \mathbb{Z}_{+}} \frac{1}{n !} \int_{\mathbb{R}^{3}} \mathrm{~d}^{3} x_{1} \int_{\mathbb{R}^{3}} \mathrm{~d}^{3} x_{2} \ldots \int_{\mathbb{R}^{3}} d^{3} x f_{n}\left(x_{1}, x_{2}, \ldots, x_{n}\right):\left|x_{1}, x_{2}, \ldots x_{n}\right\rangle\right)= \\
& =\sum_{n \in \mathbb{Z}_{+}} \frac{1}{n !} \int_{\mathbb{R}^{3}} \mathrm{~d}^{3} x_{1} \int_{\mathbb{R}^{3}} \mathrm{~d}^{3} x_{2} \ldots \int_{\mathbb{R}^{3}} \mathrm{~d}^{3} x \mathscr{H}_{\beta}^{(n)} f_{n}\left(x_{1}, x_{2}, \ldots, x_{n}\right):\left|x_{1}, x_{2}, \ldots x_{n}\right\rangle,
\end{aligned}
$$

where $f_{n} \in L_{2}^{(\mathrm{sym})}\left(\mathbb{R}^{3 n} ; \mathbb{C}\right), n \in \mathbb{Z}_{+}$, and we put, by definition,

$$
\left|x_{1}, x_{2}, \ldots x_{n}\right\rangle:=\prod_{j=1}^{n} \psi^{+}\left(x_{j}\right)|0\rangle
$$

the independent orthogonal states in the Fock space $\Phi$, generated by a cyclic vacuum vector $|0\rangle \in \Phi$, satisfying the annihilation condition $\psi(x)|0\rangle=0$ for all $x \in \mathbb{R}^{3}$.

Having constructed the corresponding $N$-particle translational-invariant Hamiltonian operators $\mathscr{H}_{\beta}^{(N)}: L_{2}^{(\mathrm{sym})}\left(\mathbb{R}^{3 N} ; \mathbb{C}\right) \rightarrow L_{2}^{(\mathrm{sym})}\left(\mathbb{R}^{3 N} ; \mathbb{C}\right)$ for arbitrary $N \in \mathbb{Z}_{+}$particles in a volume $\Lambda \subset \mathbb{R}^{3}$, one can study its a priori positive spectrum $\sigma\left(\mathscr{H}_{\beta}^{(N)}\right) \subset \mathbb{R}_{+}$and its peculiarities as a function of the density

$$
\bar{\rho}=\operatorname{tr}\{\mathscr{P} \rho(x)\}=\left.\frac{1}{\mathrm{i}} \frac{\delta \mathscr{L}(f)}{\delta f(x)}\right|_{f=0}
$$

and the inverse temperature parameter $\beta \in \mathbb{R}_{+}$. In particular, the condition 4.17) allows one to determine the above introduced measure $\mathrm{d} \mu_{\beta}$, entering the functional 4.10).

Now, it is important to recall that an equilibrium many-particle statistical system is stable [17, 18], if its generating functional satisfies the well Kubo-Martin-Schwinger analycity condition. This condition, in particular, imposes a strong analytical dependence on the inverse temperature parameter $\beta \in \mathbb{R}_{+}$of the 
spectrum $\sigma\left(\mathscr{H}_{\beta}^{(N)}\right) \subset \mathbb{R}_{+}$as $N \rightarrow \infty$ in such a way that the density $\bar{\rho}=\lim N / \Lambda \in \mathbb{R}_{+}$persists to be constant. One obtains another inference from the important fact that the number operator $\mathbf{N}_{\beta}:=\int_{\mathbb{R}^{3}} \mathrm{~d}^{3} x \rho(x)$ is a conserved quantity, that is

$$
\left[\tilde{\mathbf{H}}_{\beta}, \mathbf{N}_{\beta}\right]=\mathbf{0}
$$

for those parameters $\beta \in \mathbb{R}_{+}$, for which the equilibrium many-particle system is stable and does not pass a phase transition.

\section{The current algebra representation aspects of the collective variables transform}

The collective variables transform [5, 6, 8-10] allows one to consequently take into account and separate two different impacts of a binary interaction potential $W:=W^{(1)}+W^{(s)}$ into the many-particle distribution functions subject to its long distance $W^{(1)}$ and short distance $W^{(s)}$ parts. Since the long distance interaction potential responds for the so-called "collective" behavior of the many-particle at a bounded inverse temperature parameter $\beta \in \mathbb{R}_{+}$, the corresponding Bogolubov generating functional (4.2) can be formally rewritten in the operational form as

$$
\begin{aligned}
\mathscr{L}(f) & =Z(f) / Z(0), \\
Z(f) & :=\exp \left[-\beta W^{(\mathrm{s})}(\delta)\right] \mathscr{L}^{(\mathrm{l})}(f),
\end{aligned}
$$

where, by definition,

$$
\mathscr{L}^{(\mathrm{l})}(f):=\exp \left[-\beta W^{(\mathrm{l})}(\delta)\right] \mathscr{L}_{0}(f) .
$$

The functional (5.2) can be quite easily calculated by means of the Fourier transform representation of the long distance interaction potential and the standard quasi-classical limit $\hbar \rightarrow 0$ :

$$
\begin{aligned}
\mathscr{L}^{(\mathrm{l})}(f)= & \lim _{\hbar \rightarrow 0} \operatorname{tr}\left\{\mathscr{P}_{0} \exp \left[-\frac{\beta}{2} \int_{\mathbb{R}^{3}} \mathrm{~d}^{3} k v^{(\mathrm{l})}(k): \rho_{k} \rho_{-k}:\right] \exp [\mathrm{i} \rho(f)]\right\} \\
= & \lim _{\hbar \rightarrow 0} \operatorname{tr}\left\{\mathscr{P}_{0} \exp \left[-\frac{\beta}{2} \int_{\mathbb{R}^{3}} \mathrm{~d}^{3} k \int_{\mathbb{R}^{3}} \mathrm{~d}^{3} x \rho(x)\right]\right. \\
& \left.\times \int_{\mathbb{C}} D(\omega) \exp \left[-\int_{\mathbb{R}^{3}} \mathrm{~d}^{3} k \frac{2 \pi^{2}}{\beta v^{(1)}(k)} \omega_{k} \omega_{-k}-\int_{\mathbb{R}^{3}} \mathrm{~d}^{3} k 2 \pi \mathrm{i} \omega_{k} \rho_{k}\right] \exp [\mathrm{i} \rho(f)]\right\} \\
= & \int_{\mathbb{C}} D(\omega) J(\omega) \lim _{\hbar \rightarrow 0} \operatorname{tr}\left(\mathscr{P}_{0} \exp \left\{\mathrm{i} \rho\left[f-2 \pi \int_{\mathbb{R}^{3}} \mathrm{~d}^{3} k \omega_{k} \exp \{\mathrm{i}\langle k, x\rangle\}-\frac{\mathrm{i} \beta}{2} \int_{\mathbb{R}^{3}} \mathrm{~d}^{3} k v^{(\mathrm{l})}(k)\right]\right\}\right) \\
= & \int_{\mathbb{R}} \mathrm{d} \mu_{\beta}^{(\mathrm{l})}(\bar{z}) \int_{\mathbb{C}^{\infty}} D(\omega) J^{(\mathrm{l})}(\omega ; \bar{z}) \exp \left(\bar{z} \int_{\mathbb{R}^{3}} \mathrm{~d}^{3} x\{\exp [\mathrm{i} f(x)]-1\} g(x ; \omega)\right),
\end{aligned}
$$

where we denoted the measure $D(\omega):=\prod_{k \in \mathbb{R}^{3}} \frac{\mathrm{i}}{2} \mathrm{~d} \omega_{k} \wedge \mathrm{d} \omega_{-k}$, the parameter $\bar{z}:=z \exp \left[-\frac{\beta}{2} \int_{\mathbb{R}^{3}} \mathrm{~d}^{3} k v^{(\mathrm{l})}(k)\right]$, and the free particle system statistical operator is equal to

$$
\mathscr{P}:=\frac{\exp \left(-\beta \mathbf{H}_{0}\right)}{\operatorname{tr} \exp \left(-\beta \mathbf{H}_{0}\right)}, \quad \mathbf{H}_{0}:=\frac{\hbar^{2}}{2 m} \int_{\mathbb{R}^{3}} \mathrm{~d}^{3} x\left\langle\nabla \psi^{+}(x), \nabla \psi(x)\right\rangle,
$$

the Fourier transform

$$
v^{(\mathrm{l})}(k)=\frac{1}{(2 \pi)^{3}} \int_{\mathbb{R}^{3}} \mathrm{~d}^{3} x W^{(\mathrm{l})}(x, y) \exp (\mathrm{i}\langle k, x-y\rangle)
$$


and the measure kernels ("Jacobian")

$$
\begin{aligned}
J(\omega) & :=\exp \left\{-\int_{\mathbb{R}^{3}} \mathrm{~d}^{3} k \frac{2 \pi^{2}}{\beta v^{(\mathrm{l})}(k)} \omega_{k} \omega_{-k}+\int_{\mathbb{R}^{3}} \mathrm{~d}^{3} k \ln \frac{\pi}{\beta v^{(\mathrm{l})}(k)}\right\}, \\
J^{(\mathrm{l})}(\omega ; \bar{z}) & :=J(\omega) \exp \left\{\bar{z} \int_{\mathbb{R}^{3}} \mathrm{~d}^{3} x g^{(\mathrm{l})}(x ; \omega)\right\}, \\
g^{(\mathrm{l})}(x ; \omega) & :=\exp \left\{-2 \pi \mathrm{i} \int_{\mathbb{R}^{3}} \mathrm{~d}^{3} k \omega_{k} \exp (\mathrm{i}\langle k, x\rangle)\right\} .
\end{aligned}
$$

The formal series expansion

$$
\begin{aligned}
J^{(\mathrm{l})}(\omega ; \bar{z})= & J(\omega) \exp \left\{-\frac{\bar{z}(2 \pi)^{2}(2 \pi)^{3}}{2} \int_{\mathbb{R}^{3}} \mathrm{~d}^{3} k \omega_{k} \omega_{-k}\right. \\
& \left.+\sum_{n \in \mathbb{Z}_{+} \backslash\{2\}} \frac{\bar{z}(-2 \pi \mathrm{i})^{n}(2 \pi)^{3}}{n !} \int_{\mathbb{R}^{3}} \mathrm{~d}^{3} k_{1} \int_{\mathbb{R}^{3}} \mathrm{~d}^{3} k_{2} \ldots \int_{\mathbb{R}^{3}} \mathrm{~d}^{3} k_{n} \prod_{j=1}^{n} \omega_{k_{j}} \delta\left(\sum_{j=1}^{n} k_{j}\right)\right\} \\
= & \exp \left\{-\int_{\mathbb{R}^{3}} \mathrm{~d}^{3} k \frac{(2 \pi)^{2}\left[\beta v^{(1)}(k)(2 \pi)^{3} \bar{z}+1\right]}{\beta v^{(1)}(k)} \omega_{k} \omega_{-k}+\ldots\right\},
\end{aligned}
$$

owing to (5.3) and (5.7), right away gives rise to the approximation of the generating functional (5.2) by means of the so called "screened" long distance potential

$$
\begin{aligned}
\bar{W}^{(\mathrm{l})}(x, y) & :=\int_{\mathbb{R}^{3}} \mathrm{~d}^{3} k \frac{v^{(\mathrm{l})}(k)}{1+v^{(\mathrm{l})}(k) \beta \bar{z}(2 \pi)^{3}} \exp (\mathrm{i}\langle k, x-y\rangle) \\
& =\int_{\mathbb{R}^{3}} \mathrm{~d}^{3} k \bar{v}^{(\mathrm{l})}(k) \exp (\mathrm{i}\langle k, x-y\rangle)
\end{aligned}
$$

under the external effect of an infinite set of oscillatory potentials:

$$
\begin{aligned}
\mathscr{L}^{(\mathrm{l})}(f)= & \int_{\mathbb{R}} \mathrm{d} \mu_{\beta}^{(\mathrm{l})}(\bar{z}) \int_{\mathbb{C}^{\infty}} D(\omega) \exp \left\{-\int_{\mathbb{R}^{3}} \mathrm{~d}^{3} k \frac{(2 \pi)^{2}}{\beta \bar{v}^{(\mathrm{l})}(k)} \omega_{k} \omega_{-k}+\ldots\right\} \\
& \times \exp \left(\bar{z} \int_{\mathbb{R}^{3}} d^{3} x\{\exp [\mathrm{i} f(x)]-1\} g^{(\mathrm{l})}(x ; \omega)\right) .
\end{aligned}
$$

Having substituted the functional expression (5.9) into (5.1) one can easily obtain the corresponding Bogolubov generating functional in the Ursell-Mayer type infinite expansion form, based on the following operator expression:

$$
\begin{aligned}
Z(f):= & \exp \left[-\beta W^{(\mathrm{s})}(\delta)\right] \mathscr{L}^{(\mathrm{l})}(f) \\
= & \exp \left[-\beta W^{(\mathrm{s})}(\delta)\right] \int_{\mathbb{R}} \mathrm{d} \mu_{\beta}^{(\mathrm{l})}(\bar{z}) \exp \left(\sum_{n \in \mathbb{Z}_{+}} \frac{\bar{z}^{n}}{n !} \int_{\mathbb{R}^{3}} \mathrm{~d}^{3} x_{1} \int_{\mathbb{R}^{3}} \mathrm{~d}^{3} x_{2} \ldots \int_{\mathbb{R}^{3}} \mathrm{~d}^{3} x_{n}\right. \\
& \left.\times \prod_{j=1}^{n}\left\{\exp \left[\mathrm{i} f\left(x_{j}\right)\right]-1\right\} g_{n}^{(\mathrm{l})}\left(x_{1}, x_{2}, \ldots, x_{n}\right)\right),
\end{aligned}
$$

where $g_{n}^{(\mathrm{l})}: \mathbb{R}^{3 n} \rightarrow \mathbb{R}, n \in \mathbb{Z}_{+}$are so-called $n$-particle "cluster" distribution functions.

Observe also that the Bogolubov type generating functional (5.3) can be rewritten in the integral Gauss 
type form as

$$
\begin{aligned}
\mathscr{L}^{(\mathrm{l})}(f) & =\int_{\mathbb{C}^{\infty} \times \mathbb{R}} \mathrm{d} \mu_{\beta}^{(\mathrm{l})}(\bar{z}, \omega) J^{(\mathrm{l})}(\omega, \bar{z}) \exp \left(\bar{z} \int_{\mathbb{R}^{3}} \mathrm{~d}^{3} x\{\exp [\mathrm{i} f(x)]-1\} g^{(\mathrm{l})}(x ; \omega)\right) \\
& =\int_{\xi \in \mathbb{C}^{\infty} \times \mathbb{R}} \mathrm{d} \bar{\mu}_{\beta}^{(\mathrm{l})}(\bar{\xi}) \mathscr{L}_{0}^{(\mathrm{l})}(f ; \xi),
\end{aligned}
$$

where, by definition, $\xi:=\left(\omega, \bar{z}, \mathbb{R}^{3}\right)$ and for any Lebesgue measurable set $A \subset \mathbb{R} \times \mathbb{C}^{\infty} \times \mathbb{R}^{3}$, the measure

$$
\bar{\mu}_{\beta}^{(\mathrm{l})}(A):=\int_{A \subset \mathbb{R} \times \mathbb{C}^{\infty} \times \mathbb{R}^{3}} \mathrm{~d}^{3} k \mathrm{~d} \mu_{\beta}^{(\mathrm{l})}(\bar{z}) D(\omega) J^{(\mathrm{l})}(\omega, \bar{z})
$$

with a reduced generating functional $\mathscr{L}_{0}^{(\mathrm{l})}(f ; \omega, \bar{z}, k),(\omega, \bar{z}, k) \in \mathbb{C}^{\infty} \times \mathbb{R} \times \mathbb{R}^{3}$, satisfying [13] the following functional equation:

$$
\left[\nabla_{x}-\nabla f(x)\right] \frac{1}{\mathrm{i}} \frac{\delta}{\delta f(x)} \mathscr{L}_{0}^{(\mathrm{l})}(f ; \omega, \bar{z}, k)=2 \pi k \omega_{k} \exp (\mathrm{i}\langle k, x\rangle) \frac{1}{\mathrm{i}} \frac{\delta}{\delta f(x)} \mathscr{L}_{0}^{(\mathrm{l})}(f ; \omega, \bar{z}, k)
$$

Hence, one can obtain the effective partial renormalized long distance Hamiltonian operator

$$
\tilde{\mathbf{H}}_{\beta}^{(l)}(\omega, \bar{z}, k):=\frac{\hbar^{2}}{8 m} \int_{\mathbb{R}^{3}} \mathrm{~d}^{3} x\left\langle\tilde{K}_{\beta}^{(l)+}(x, \omega, \bar{z}, k), \rho^{-1}(x) \tilde{K}_{\beta}^{(l)}(x, \omega, \bar{z}, k)\right\rangle,
$$

where we put, by definition,

$$
\tilde{K}_{\beta}^{(l)+}(x, \omega, \bar{z}, k):=K(x)-2 \pi k \omega_{k} \rho(x) \exp (\mathrm{i}\langle k, x\rangle)
$$

for all $x \in \mathbb{R}^{3}$ and $(\omega, \bar{z}, k) \in \mathbb{C}^{\infty} \times \mathbb{R} \times \mathbb{R}^{3}$. As a result of [5.14), one finds [13] that the effective external long distance oscillatory potential

$$
\tilde{W}_{\beta}^{(\mathrm{l})}(x ; \omega, \bar{z}, k)=\frac{\hbar^{2} \pi \omega_{k} k^{2}}{2 m} \exp (\mathrm{i}\langle k, x\rangle)\left[1+\pi \omega_{k} k \exp (\mathrm{i}\langle k, x\rangle)\right]
$$

yields the following partial canonical Hamiltonian operator expression:

$$
\tilde{\mathbf{H}}_{\beta}(\omega, \bar{z}, k)=\frac{\hbar^{2}}{2 m} \int_{\mathbb{R}^{3}} \mathrm{~d}^{3} x\left\langle\nabla \psi^{+}(x), \nabla \psi(x)\right\rangle+\int_{\mathbb{R}^{3}} \mathrm{~d}^{3} x \tilde{W}_{\beta}^{(\mathrm{l})}(x ; \omega, \bar{z}, k) \psi^{+}(x) \psi(x),
$$

whose generating functional of the current group $G=\mathscr{S} \rtimes \operatorname{Diff}\left(\mathbb{R}^{3}\right)$ equals the expression (5.11). Moreover, taking into account the representation (5.10), one can construct the full effective long distance Hamiltonian operator

$$
\tilde{\mathbf{H}}_{\beta}^{(\mathrm{l})}=\frac{\hbar^{2}}{8 m} \int_{\mathbb{R}^{3}} \mathrm{~d}^{3} x\left\langle\tilde{K}_{\beta}^{(l)+}(x ; \rho), \rho^{-1}(x) \tilde{K}_{\beta}^{(\mathrm{l})}(x ; \rho)\right\rangle,
$$

where, by definition, we put

$$
\begin{aligned}
\tilde{K}_{\beta}^{(l)+}(x ; \rho):= & K(x)-\sum_{n \in \mathbb{Z}_{+}} \frac{1}{(n-1) !} \int_{\mathbb{R}^{3}} \mathrm{~d}^{3} x_{1} \int_{\mathbb{R}^{3}} \mathrm{~d}^{3} x_{2} \ldots \int_{\mathbb{R}^{3}} \mathrm{~d}^{3} x_{n} \\
& \times \nabla_{x} \ln g_{n}^{(\mathrm{l})}\left(x_{1}, x_{2}, \ldots x_{n}\right): \rho\left(x_{1}\right) \rho\left(x_{2}\right) \ldots \rho\left(x_{n}\right): .
\end{aligned}
$$

As a simple corollary from the expression (5.18), one obtains that the effective long-distance Hamiltonian operator contains an infinite hierarchy of multinary potential energy terms, which should be in due course taken into account when studying the peculiarities of the corresponding many-particle distribution functions. In particular, the energy spectrum of the $N$-particle canonical representation of the 
Hamiltonian operator (5.18) possesses an important information on the many-particle system stability at a special inverse temperature parameter $\beta \in \mathbb{R}_{+}$.

As an example demonstrating the effective Hamiltonian operator construction, we will consider a one-dimensional many-particle system of density $\bar{\rho} \in \mathbb{R}_{+}$on an axis $\mathbb{R}$ at a finite inverse temperature $\beta \in \mathbb{R}_{+}$, described by the following operator expression in the canonical Fock space $\Phi$ :

$$
\mathbf{H}=\frac{\hbar^{2}}{2 m} \int_{\mathbb{R}} \mathrm{d} x\left\langle\nabla \psi^{+}(x), \nabla \psi(x)\right\rangle-\lambda \int_{\mathbb{R}} \mathrm{d} x \int_{\mathbb{R}} \mathrm{d} y \ln |x-y| \psi^{+}(x) \psi^{+}(y) \psi(y) \psi(x),
$$

where $\lambda \in \mathbb{R}_{+}$is a positive parameter. The corresponding Bogolubov generating functional $\mathscr{L}(f), f \in$ $\mathscr{S}(\mathbb{R} ; \mathbb{R})$, satisfies, owing to 4.12 ), the following functional equation:

$$
\left[\nabla_{x}-\mathrm{i} \nabla f(x)\right] \frac{1}{\mathrm{i}} \frac{\delta \mathscr{L}(f)}{\delta f(x)}=2 \lambda \beta \int_{\mathbb{R}} \mathrm{d} y \nabla_{x} \ln |x-y|: \frac{1}{\mathrm{i}} \frac{\delta}{\delta f(x)} \frac{1}{\mathrm{i}} \frac{\delta}{\delta f(x)}: \mathscr{L}(f) .
$$

Taking now into account that the expression

$$
A(x ; \rho)=2 \lambda \beta \int_{\mathbb{R}} \mathrm{d} y \nabla_{x} \ln |x-y|: \rho(x) \rho(y):
$$

one can easily construct the effective renormalized Hamiltonian operator

$$
\tilde{\mathbf{H}}_{\beta}:=\frac{\hbar^{2}}{8 m} \int_{\mathbb{R}} \mathrm{d} x\left\langle\tilde{K}_{\beta}^{+}(x ; \rho), \rho^{-1}(x) \tilde{K}_{\beta}(x ; \rho)\right\rangle,
$$

acting in a suitable Hilbert space $\mathscr{H}_{\beta}$, realizing a non-reducible representation of the basic current group $G=\mathscr{S} \rtimes \operatorname{Diff}(\mathbb{R})$. It is quite easy to calculate the resulting effective Hamiltonian operator (5.23) expression in the canonical Fock space $\Phi$ :

$$
\tilde{\mathbf{H}}_{\beta}=\frac{\hbar^{2}}{2 m} \int_{\mathbb{R}} \mathrm{d} x\left\langle\nabla \psi^{+}(x), \nabla \psi(x)\right\rangle+\frac{\hbar^{2}}{2 m} \int_{\mathbb{R}} \mathrm{d} x \int_{\mathbb{R}} \mathrm{d} y \frac{\lambda \beta(\lambda \beta-1)}{|x-y|^{2}} \psi^{+}(x) \psi^{+}(y) \psi(y), \psi(x),
$$

describing an infinite set of particles on the axis $\mathbb{R}$, binarily interacting to each other by means of the inverse square potential

$$
\tilde{W}_{\beta}(x, y):=\frac{\hbar^{2} \lambda \beta(\lambda \beta-1)}{2 m|x-y|^{2}}
$$

where $x \neq y \in \mathbb{R}$ and $\lambda, \beta \in \mathbb{R}_{+}$are suitable positive parameters. Here, it is necessary to mention that the effective Hamiltonian operator (5.24) realizes a nonreducible representation of the current group $G=\mathscr{S} \rtimes \operatorname{Diff}(\mathbb{R})$ in the Hilbert space $\mathscr{H}_{\beta}$, generated by its ground cyclic eigenstate $\Omega_{\beta} \in \mathscr{H}_{\beta}$, satisfying the determining condition [2.17). It can be shown [11, 13, 14] that

$$
\bar{\varepsilon}_{\beta}:=\inf _{\omega \in \mathscr{\mathcal { X }}_{\beta},\|\omega\|=1} \frac{\left(\omega, \tilde{\mathbf{H}}_{\beta} \omega\right)}{\left(\omega, \tilde{\mathbf{N}}_{\beta} \omega\right)}=\lambda^{2} \beta^{2} \pi^{2} \bar{\rho}^{2} / 6
$$

holds, where we denoted by $\tilde{\mathbf{N}}_{\beta}:=\int_{\mathbb{R}} \mathrm{d} x \rho(x)$ the corresponding particle number operator in the Hilbert space $\mathscr{H}_{\beta}$. The least average energy per particle (5.26) analytically depends on the inverse temperature parameter $\beta \in \mathbb{R}_{+}$. The same can also be obtained for the other energy excitations of the Hamiltonian operator (5.24). Thus, we infer that the initial one-dimensional many-particle system with the Hamiltonian (5.20) and at the inverse temperature parameter $\beta \in \mathbb{R}_{+}$is completely stable and permits no phase transition. Moreover, at the temperature parameter $\beta=1 / \lambda \in \mathbb{R}_{+}$the effective Hamiltonian operator (5.24) describes a many-particle noninteracting system of the density $\bar{\rho} \in \mathbb{R}_{+}$and the least average energy per particle $\bar{\varepsilon}_{\beta}=\pi^{2} \bar{\rho}^{2} / 6$, depending only on the density. 


\section{Conclusion}

The investigation of statistical properties of classical many-particle systems at a finite inverse temperature $\beta \in \mathbb{R}_{+}$and a fixed density $\bar{\rho} \in \mathbb{R}_{+}$by means of the current algebra representations has two main reasons: firstly, it provides an interesting reformulation of the initial quantum statistical problem in terms of physical observables such as the particle number density and the particle flux density, rather than the corresponding second-quantized field creation and annihilation operators.

The second reason is related to a very rich structure of the current group $G=\mathscr{S} \rtimes$ Diff( $\left(\mathbb{R}^{3}\right)$ irreducible representations, according to the Bogolubov functional equation for the generating many-particle distribution functional, and whose analytical property subject to the temperature parameter $\beta \in \mathbb{R}_{+}$are responsible for the system stability as it follows from the Kubo-Martin-Schwinger approach, applied to the classical statistical mechanics.

Moreover, a very rich functional-operator structure of solutions to the related Bogolubov functional equations allows one to make physically reasonable re-expansions of the general irreducible representation measure, as it was show for the case of the classical collective variables transform, and whose generating functional permits an additive Gauss type representation, based on an infinite set of free noninteracting many-particle systems embedded in an external oscillatory type potential field.

As a dual aspect of irreducible representations of the current group $G=\mathscr{S} \rtimes \operatorname{Diff}\left(\mathbb{R}^{3}\right)$, related to the Bogolubov functional equation, we need to mention the construction of associated effective Hamiltonian operators subject to the basic ground state cyclic representation of the current group, whose analytical properties are responsible for the many-particle system stability and possibly, for the phase transition behavior. We hope that the approach devised in the work will prove to be helpful in further gaining insight into the statistical clustering properties of many-particle systems and in developing new more powerful and specialized analytical techniques for solving other interesting problems in statistical physics.

\section{Acknowledgements}

Authors are cordially thankful to prof. J. Sławianowski and prof. J. Spałek for interesting discussions, important comments and remarks.

\section{References}

1. Araki H., Publ. RIMS, Kyoto University, 1969/70, 5, 361-422.

2. Arefyeva I.Ya., Teor. Mat. Fiz., 1972, 10, No. 2, 223-237 (in Russian).

3. Carey A.L., Hannabuss K.C., J. Funct. Anal., 1987, 75, 128-160; doi 10.1016/0022-1236(87)90109-1.

4. Newman Ch.M., Commun. Math. Phys., 1972, 26, No. 3, 169-204; doi 10.1007/BF01645089

5. Bohm D., The General Collective Variables Theory, Moscow, Mir, 1964 (in Russian).

6. Bogoliubov N.N., Problems of Dynamic Theory in Statistical Physics, OGIZ, Gostekhizdat, Moscow, 1946 (in Russian) [Bogoliubov N.N., Problems of Dynamic Theory in Statistical Physics, Technical Information Service, Oak Ridge, Tennessee, 1960].

7. Zubarev D.N., DAN SSSR, 1954, 95, No. 4, 757-760 (in Russian).

8. Yukhnovskii I.R., Holovko M.F., Statistical Physics of Equilibrium Systems, Kyiv, Naukova Dumnka, 1980 (in Russian).

9. Bogolubov N.N. (Jr.), Prykarpatsky A.K., Teor. Mat. Fiz., 1986, 66, No. 3, 463-480 (in Russian).

10. Prykarpatsky A.K., DAN AN SSSR, 1985, 285, No. 5, 1096-1101 (in Russian).

11. Bogolubov N.N. (Jr.), Prykarpatsky A.K., Phys. Part. Nuclei, 1986, 17, No. 4, 789-920 (in Russian)

12. Vladimirov V.S., Generalized Functions in Mathematical Physics, Moscow, Nauka, 1979, (in Russian).

13. Goldin G.A., Menicoff R., Sharp D.H., J. Math. Phys., 1980, 21, No. 4, 650-664; doi $10.1063 / 1.524510$.

14. Goldin G.A., Grodnik J., Powers R.T., Sharp D.H., J. Math. Phys., 1974, 15, No. 1, 88-100; doi 10.1063/1.1666513

15. Bogolubov N.N., Bogolubov N.N. (Jr.), Introduction into Quantum Statistical Mechanics, World Scientific, New Jersey, 1986.

16. Bogolubov N.N., Shirkov D.V., Introduction to the Theory of Quantized Fields, Interscience, New York, 1959.

17. Aizenman M., Goldstein S., Gruber C., Lebowitz J.L., Martyin P., Commun. Math. Phys., 1977, 53, 209-220; doi $10.1007 / \mathrm{BF} 01609847$ 


\title{
Підхід до класичної рівноважної статистичної механіки на основі алгебри струмів та його застосування
}

\author{
М.М. Боголюбов (мол. $)^{11}$, А.К. Прикарпатський리 \\ ${ }^{1}$ Математичний інститут ім. В.А. Стєклова РАН, Москва, Росія \\ 2 Академія гірництва та металургії, Краків, Польща \\ 3 Державний педагогічний університет ім. І. Франка, Дрогобич, Україна
}

Аналізується підхід до вивчення функцій розподілу систем багатьох частинок при рівноважній температурі та властивості їх стабільності, що ґрунтується на представленнях нерелятивістичної алгебри струмів. Показано, що метод породжуючого функції розподілу класичного функціоналу Боголюбова $\epsilon$ досить ефективним інструментом для побудови незвідних представлень алгебри струмів та відповідних узагальнених розкладів мір, включаючи відоме перетворення до колективних змінних. Запропонована конструкція ефективного оператора Гамільтона, обговорюються особливості його спектра в залежності від стійкості рівноваги систем багатьох частинок.

Ключові слова: алгебра струмів, породжуючий функціонал Боголюбова, представлення колективних змінних, реконструкція оператора Гамільтона 


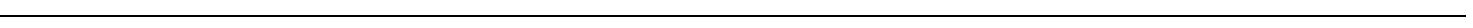

